\section{Tipping the energy balance}

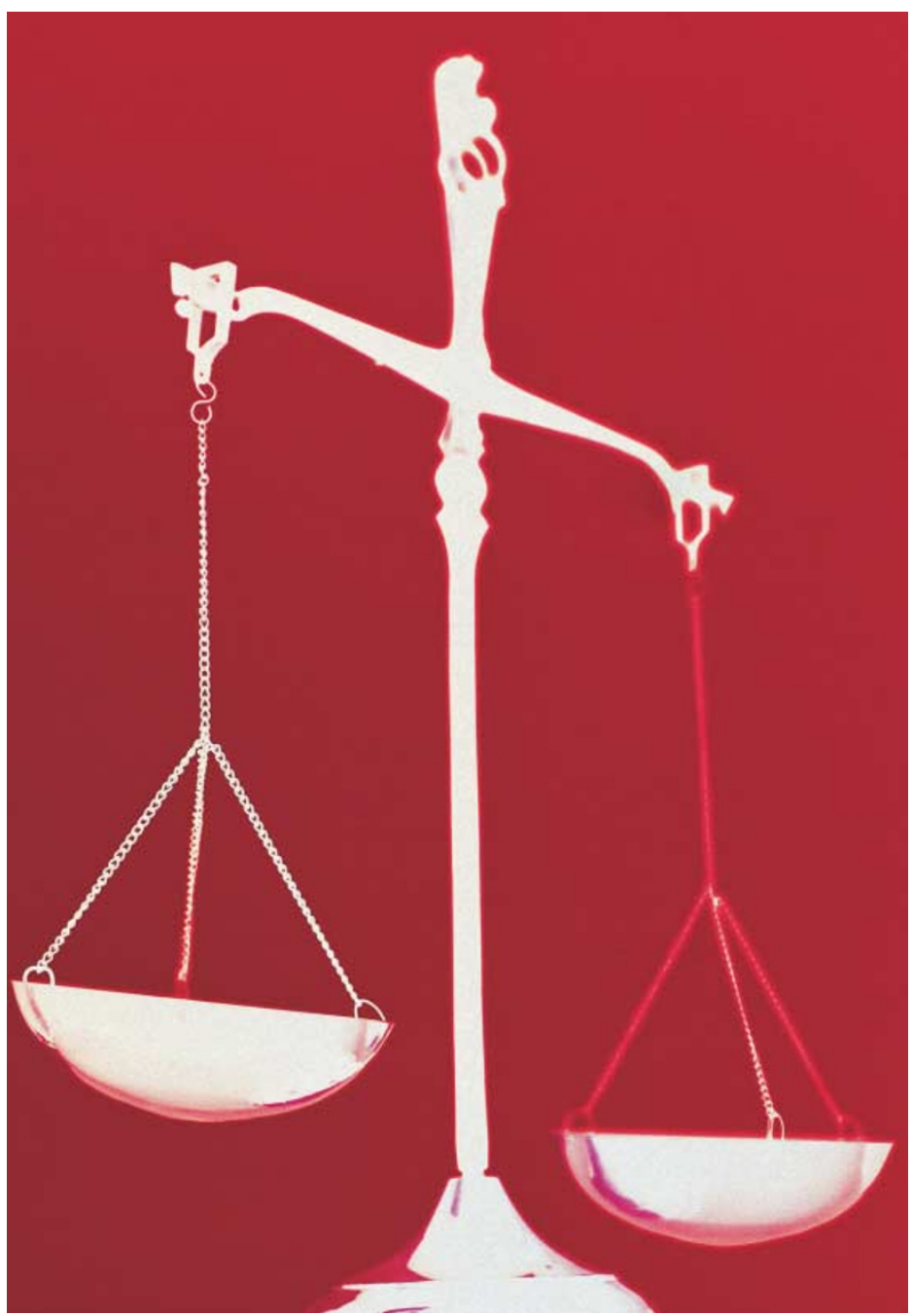

Those of us with physical evidence of a few dietary indulgences might well be advised to climb some stairs and put a lock on the biscuit tin. But for the increasing number of people worldwide for whom excess weight is more than just an aesthetic problem, the most promising remedy will probably come as a pill that either curbs the appetite or prevents weight gain. By creating two mouse mutants that do not pile on the pounds, two groups now report progress towards producing such a wonder drug.

The first study started with a lean mutant mouse. Animals in which $C / e b p \alpha$, one of several genes required for the differentiation of fat cells, is replaced with another such energy to burning it. The number of active mitochondria is increased in $\beta / \beta$ WAT, probably due to increased levels of the $G$ protein $\alpha$ stimulatory protein $\left(\mathrm{G} \alpha_{\mathrm{s}}\right)$ in WAT; this hypothesis, which was based on gene expresssion levels, was confirmed when overexpression of $\mathrm{G} \alpha$ led to the production of new mitchondria in fat-laden cells in culture.

A second study looked at another aspect of defects in regulating energy balance. By placing stress on the body, obesity leads to several health problems, one of which is the development of insulin-resistant (type 2) diabetes. Mouse studies have highlighted the activation of the JNK signalling pathway in obesity and insulin resistance, and Anja Jaeschke and co-workers have looked into this in more detail. To obviate the pleiotropic effects of removing JNK itself, the authors have analysed mice that are mutant for Jip1, which encodes a scaffolding protein downstream in this pathway. Like the $\beta / \beta$ mice, Jip $1^{-/}$animals didn't gain weight when put on a high-fat diet; in addition, they showed increased insulin sensitivity in adipose tissue. Jip1 deficiency therefore protects mice against obesity and insulin resistance. The way it does so is by reducing the activation of the JNK pathway in fat and muscle in response to high food intake, which in turn increases an inhibitory signal to the insulin pathway.

Harnessing the weight-busting power of $\mathrm{G} \alpha_{\mathrm{s}}$ and of Jip1 mutants is what dreams are made of. But are they too good to be true? JIP1 is a candidate gene for type 2 diabetes in humans, so the prospects of transposing the studies from mouse to man - leading ultimately to drugs that block JIP1 - look promising. The therapeutic value of understanding the phenotype of the $\beta / \beta$ mouse was also emphasized by the observation that the $\beta / \beta$ knock-in alleles are able to mitigate the obese phenotypes of two mouse models, Lep ${ }^{o b}$ and $C p e^{f a t}$, which gain weight through excessive eating or lowered metabolism, respectively. The two studies combined therefore put us in good stead to counter both environmental and hereditary causes of obesity, and its damaging consequences on health.

(2) References and links Tanita Casci

\section{IN THE NEWS}

Gene therapy for simian slackers

In a study guaranteed to capture the imagination of any boss with a less than enthusiastic workforce, scientists in the United States have succeeded in turning lazy monkeys into workaholics by turning off a single gene.

"Normal monkeys and people procrastinate - tend not to work very well when they have a lot of time to get the job done, and work better when the reward is nearer in time," explained Barry Richmond, lead researcher of the study, published in the journal Proceedings of the National Academy of Sciences (BBC News Online)

To change this behaviour, Richmond's group used an antisense construct injected into the brain to block the expression of a receptor that normally sensitizes cells to dopamine, a neurotransmitter involved in reward perception. The monkeys were then set to work on a task in which the eventual reward was a long way off.

Once expression of the gene was blocked, the monkeys were no longer able to perceive how much work they would have to do to receive a reward and performed the task much more diligently. "The monkeys became extreme workaholics, as evidenced by a sustained low rate of errors [...] irrespective of how distant the reward might be", commented Richmond, "This was conspicuously out of character for these animals" (Independent Online).

Reassuringly, these results are more likely to be used in understanding mental illness than in boosting productivity in the workplace: "...the ability to associate work with reward is disturbed in mental disorders, including schizophrenia, mood disorders and obsessivecompulsive disorder, so our finding of the pivotal role played by this gene and circuit may be of clinical interest", Richmond concluded (Seattle Times).

Louisa Flintoft 